\title{
Barriers and Solutions for Improving Pain Management Practices in Acute Hospital Settings: Perspectives of Healthcare Practitioners for a Pain-Free Hospital Initiative
}

\author{
Nuraqilah Akbar ${ }^{1}$, Shyh Poh Teo ${ }^{2}$, Hjh Noor Artini Hj-Abdul-Rahman ${ }^{2}$, Hjh Asmah Hj-Husaini ${ }^{1}$, \\ Munikumar Ramasamy Venkatasalu ${ }^{3}$ \\ ${ }^{1}$ Pengiran Anak Puteri Rashidah Sa'adatul Bolkiah (PAPRSB) Institute of Health Sciences, Universiti Brunei Darussalam, Brunei Darussalam \\ ${ }^{2}$ Geriatrics and Palliative Unit, Department of Internal Medicine, Raja Isteri Pengiran Anak Saleha (RIPAS) Hospital, Brunei Darussalam \\ ${ }^{3}$ Faculty of Health and Life Sciences, Oxford Brookes University, Oxford, UK
}

Corresponding Author:

Shyh Poh Teo, FRACP

Geriatrics and Palliative Unit,

Department of Internal Medicine, Raja

Isteri Pengiran Anak Saleha (RIPAS)

Hospital, Brunei Darussalam.

E-mail: shyhpoh.teo@moh.gov.bn

ORCID:

https://orcid.org/0000-0002-6117-5774

Received: November 2, 2019

Revised: November 27, 2019

Accepted: December 3, 2019

\begin{abstract}
Background: Effective pain management is a fundamental human right. However, global disparities in pain management practices exist across health settings. This study explored healthcare practitioners' views on pain management in the acute care hospital setting. Methods: The focus groups included clinical specialties most likely to encounter patients with 'difficult to manage pain', namely those in the Geriatrics and Palliative Care Unit (2 doctors and 3 nurses), Critical Care Unit (7 doctors), and the Pain Management Team (3 doctors and 2 nurses). The transcripts were analyzed using a qualitative thematic analysis. Results: The data analysis revealed four themes. Theme 1, 'Being too safe' described the presence of apprehensive attitudes among patients and healthcare practitioners that limits the appropriate use of diverse and tailored pain medications in acute care hospital settings. Theme 2, 'Working as a team' described the need for collaborative approaches to achieve hospital-wide evidence-based pain management. Theme 3, 'Adaptation for local and cultural preferences' explored how pain was perceived through cultural lenses and suggested strategies to tailor pain management to local and cultural preferences. Finally, Theme 4, 'Driving acute pain management forward' listed clinician solutions for improving pain management in acute care hospital settings toward a pain-free hospital initiative. Conclusion: Despite advances in pain medicine and pain teaching strategies, effective pain management is proportionate to both clinical and cultural preferences. Future studies should investigate the standardization of global pain management tools and guidelines to fit the local culture and context.
\end{abstract}

Key Words: Acute pain, Pain clinics, Pain management, Symptom assessment, Hospitals

\section{INTRODUCTION}

Pain is a common condition encountered by healthcare professionals, especially those providing care for older patients. ${ }^{1)}$ Pain is associated with significant disability, reduced mobility, falls, anxiety, depression, and social isolation. ${ }^{1,2)}$ In addition, pain is a frequent complication of patients admitted to hospitals and negatively impacts multiple aspects of health, including the development of chronic pain. ${ }^{3)}$ Pain is poorly managed for reasons ranging from clinicians' attitudes of focusing on pathophysiology rather than the quality of life to cultural, societal, and organizational reasons. However, pain management is a fundamental human right; thus, strategies and efforts are required to improve pain. ${ }^{4)}$

A review of quality monitoring data from 8 large hospitals in the United States identified the following 6 quality indicators for optimal pain management in a hospital setting: documented pain in- 
tensity using a numeric or descriptive rating scale; documented pain intensity at frequent intervals; treatment of pain other than by the intramuscular route; treatment with regular analgesia; and if possible, a multimodal approach of pain prevention and control to facilitate function and quality of life; and the education and knowledge of patients regarding pain management. ${ }^{5)}$ However, diverse factors such as fears related to opioid side effects, communication issues, cultural beliefs, and lack of knowledge limit effective pain management. ${ }^{6)}$

Pain-free hospital initiatives that integrate pain treatment into routine hospital care have been implemented internationally to achieve pain management quality indicators. For example, in Poland, hospitals that maintain high standards of postoperative analgesia in terms of preoperative patient information, assessment and documentation, and monitoring of medication side effects and complications are certified as 'pain-free hospitals'. These hospitals provide positive outcomes for both patients and the hospitals, with improved recovery, reduced complications, shorter hospital stays, and improved patient satisfaction. ${ }^{7}$

Raja Isteri Pengiran Anak Saleha (RIPAS) Hospital, the main tertiary hospital in Brunei Darussalam with 880 beds, strives to improve hospital pain management. Currently, the hospital's Pain Management Team focuses on postoperative management, while other teams are responsible for managing pain in their respective patients. To implement measures to ensure consistent pain assessment and management throughout the hospital, it is crucial to understand the current pain assessment and management practices. Therefore, the present study explored the views of clinicians regarding acute pain management in the hospital setting to move toward a pain-free hospital.

\section{MATERIALS AND METHODS}

Health professionals from three clinical specialties most likely to encounter patients with 'difficult to manage pain', namely the Geriatrics and Palliative Care Unit, the Critical Care Unit, and the Pain Management Team, were invited to participate in the study. The different views and perspectives of these specialties were expected to contribute toward a better understanding of barriers within the hospital. The inclusion criteria for the participants were health professionals who worked in the hospital for at least 1 year, who were involved in pain management within their scope of practice, and who were able to speak good English. The participants were informed about the study by the heads of each department. Potential participants who required more details regarding the study or who agreed to participate were contacted by the researchers.

A qualitative approach was utilized to gain insight into the prob- lems experienced by the participants regarding acute pain management in hospital settings. Focus groups were used to determine the participants' views on issues related to acute pain management, as this approach allowed researchers to elicit a large amount of rich data from different perspectives over a set time. ${ }^{8)}$ The semi-structured, in-depth focus groups lasted between 30 and 60 minutes and were both audio-recorded and transcribed verbatim. The transcripts were analyzed using qualitative thematic analysis. An open coding process was performed independently by the researchers (NA, MV, and AH). The generated codes were discussed, and after a series of inductive analyses with the team members, the codes that conveyed similar meanings were thematically clustered into categories.

Written informed consent was obtained from the participants before inclusion in each focus group. This study was approved by the joint Pengiran Anak Puteri Rashidah Sa'adatul Bolkiah (PAPRSB) Institute of Health Sciences Research Committee and the Medical and Health Research and Ethics Committee, Ministry of Health, Brunei Darussalam (No. UBD/IHS/B3/8).

\section{RESULTS}

Three separate focus groups were formed with 17 participants in total: the Geriatrics and Palliative Care Unit ( 2 doctors and 3 nurses), the Critical Care Unit (7 doctors), and the Pain Management Team ( 3 doctors and 2 nurses). The analysis revealed four themes: being too safe, working as a team, adaptation for local and cultural preferences, and driving acute pain management forward.

\section{Theme 1: Being Too Safe}

This theme described the presence of apprehensive attitudes among patients and healthcare practitioners due to complex pain scenarios, which prevent the appropriate use of diverse and tailored pain medications across acute hospital settings. The participants understood pain as a complex phenomenon requiring indepth knowledge and understanding. Optimal management required an ability to consider the patient's condition and health circumstances. Participant \#7 described the multifaceted considerations faced by clinicians before prescribing analgesia as follows:

"When you prescribe any pain medication, you have to look at the health of the patient... if the patient has systemic disease, then it will be different. If the patient is elderly... Does the patient have renal disease? Does the patient have lung disease? Does the patient have allergies?" (P7)

To take the patients' health care needs into account, clinicians 
need to constantly update their knowledge regarding pain management. Inadequate experience or training may lead to apprehension regarding medications and side effects. Apprehension regarding the potential side effects of pain medications may lead clinicians to prescribe lower doses of drugs or less efficacious medications as safer practice, which may impact adequate patient pain relief:

"They are afraid of the side effects; they don't know how to treat the side effects. So they usually give the same stuff... Paracetamol, Tramadol, maybe pethidine, but they won't give the strongest stuff. They don't know much about diverse drugs; therefore it is safer for them not to give." (P5)

Issues regarding patients' understanding of pain management, particularly their expectations of the benefits and side effects of treatment were also identified. Clinicians' perceptions of their patients' understanding of their pain management plan could influence their approach:

"Some patients don't like Norgesic, because they say it makes them 'pening' (dizzy). But they don't realize that pethidine will make them more 'pening.'” (P10)

Participants also expressed concerns regarding the risks of harm due to patients misunderstanding their pain management plan. These patient care experiences resulted in extra care when providing advanced pain management techniques, particularly those requiring self-management by patients:

"I just worry about the lack of knowledge of patients because I know if they have a syringe driver, and if they are a bit anxious, they'll probably ramp it up. Just like we had patients with pneumonia, they bought an oxygen machine. And you ask ... 'Don't you think about checking what's the problem.'” (P2)

These common uncertainties faced by participants led them to express the urgent need for a strategy to raise awareness of safe pain management within patients and the local community to achieve a standard approach, with mutual understanding and expectations from both clinicians and patients. One participant stated that some wards had no educational activities to promote patients' understanding of their pain awareness:

"We hope that they (patients) are given the information because we do have information leaflets regarding that (postoperative pain). Some wards are better than others, some zilch, totally nothing." (P9)
In contrast, the participants suggested a standard operating procedure (SOP) outlining ways to manage patients' safety concerns:

"We need to have a policy or SOP. How do we make sure there is no abuse? How to make sure the law governs the giver and receiver..."

\section{Theme 2: Working as a Team}

This theme described the importance of leadership and teamwork in pain management in hospitals, which can be influenced by the willingness of senior practitioners to apply evidence-based pain management and the sense of leadership in managing patients' pain as a team.

The participants described 'hierarchical challenges' in the hospital that impeded evidence-based pain management practices in acute hospital settings. Although pain knowledge and understanding have improved over time, clinicians may be unwilling to change their pain management strategies:

"Every year, I give them tutorial(s) on acute pain management... but when you go back to your ward, you follow what your seniors do. They learn, but when they see senior doctors, the boss says so, you follow." (P10)

\section{Communicating patient's pain between staff}

Good communication is vital to ensure clinical assessment and treatment of patients complaining of pain. Handover between staff regarding pain and communicating patients' needs require further improvement to ensure continuity of care. The participants reported that staff compensate for limited handovers by duplicating pain history-taking and assessment:

"Doctors are not there all the time, nurses are. And the message has to reach the doctor from the nurses. Even epidural analgesia, they (patients) tell the midwife, the midwife (should) tell the anesthetist." (P10)

"First of all, the pain nurse will talk to them, and then when the anesthetist goes to see them, they will ask again." (P4)

\section{Collaborative approach within a multidisciplinary team}

The participants agreed that health professionals should work together toward improving pain management. As this is a shared responsibility among clinicians, a collaborative approach within a multidisciplinary team is required within the hospital:

"It's not just us who has to assess, our nurses will give feedback. Our physio(therapist)s and OTs (Occupational Therapists) will also give 
feedback. Physio would say 'When they put weight on that side, we think the knee is sore. Can you look at that?' And we may not have assessed that well when they are lying in bed in the morning. Same with occupational therapy, when dressing or transferring, they will mention (pain) to us." (P2)

The participants also included family members as part of the "team" involved in pain assessment and management:

"Health professionals know how to assess pain. We can involve family to assess the pain. So there is no specific... which we call pain nurse, pain team." (P3)

\section{Theme 3: Adaptation for Local and Cultural Preferences}

This theme explored how pain was perceived through cultural lenses and proposed strategies to tailor pain management to local preferences.

\section{Cultural barriers in assessment}

The participants felt that cultural variations should be considered during pain assessment and management. The language used in standard pain assessment tools to assess pain severity may not apply well in local settings:

"We tend to use more descriptive (terms) and the thing I use is how it affects their daily activities in life. Does it affect their sleep, does it affect their eating, does it affect their moving? If it affects them, it's quite severe pain. Cause for them, they don't understand what the tools mean." (P1)

The participants also felt that patients were not as forthcoming when asking for analgesia, possibly due to cultural reasons. Patient requests for pain relief were considered an indication of high-intensity or severe pain:

"Bruneians have got a very high pain threshold. So we don't need much pain relief. They are polite people. So if they are in pain, they endure. So they don't even ask the nurse." (P10)

Incorporating cultural preferences in pain management

The participants also considered cultural preferences when providing pain management. The majority of the population are Muslim and religion seemed to influence the effectiveness of the pain management strategy. The participants appeared to embrace this culture but were less confident in suggesting that their patients incorporate their religious values into pain management:

"Use the zikir (prayers) to recite in silence. It depends on their beliefs but it actually helps. But I don't know how to reinforce this non-pharmacological (approach) from the Islamic perspective." (P5)

Family involvement is also a cultural norm that influences the success of pain management:

"We ask family members to sit by their side to calm them. Some patients want family members; people they are familiar with to comfort them." (P1)

\section{Theme 4: Driving Acute Pain Management Forward}

Clinician buy-in

'Clinician buy-in' and support is required from all relevant stakeholders to drive pain management forward. The barriers and different perspectives that may hinder pain-free hospital initiatives should be considered and discussed with stakeholders before initiating changes. This belief was illustrated in the following statements:

"If you want to introduce something, it means more work for the nurses. It means more work for the recovery (staff) and then, of course, they are resistant to do it. And we can't blame them because they are overloaded as well." (P7)

"I was flagged up so many impossibilities. 'Patient is going to be nil by mouth', I said 'Give suppository', 'They don't have any suppositories available'. 'Get some from OT (operating theatre)'. 'Oh, sometimes OT won't give it'. The patient is in pain, you know." (P9)

The participants indicated that improving pain management would be challenging without teamwork and agreement from all relevant parties.

\section{Guidelines and mentorship}

The participants also felt that localized guidelines would be useful to ensure a consistent clinical approach. Informally, staff have adapted or referred to those available overseas:

"In terms of a localized guideline, whether adapted or informal, I can simply say no such guideline. In terms of practice, we refer to management (guidelines) from the United Kingdom. If we look at all these guidelines, there are some differences. We will just agree and adapt to the local (situation)." (P1)

They also felt that mentorship is required to guide and train staff in the improvement in pain management:

"When I first started, it's just Panadol (Paracetamol), nothing or Panadol. 'What about tramadol? or 'Maybe we'll just try a little bit of 
Morphine'. Now our junior team is more confident with use of opioids. And pharmacological treatment is a bit more optimal." (P2)

Another participant suggested the need for mentors or champions throughout the hospital to achieve the goal of a pain-free hospital:

"There should be someone in each ward, who is perhaps a pain lead. They don't have to (be) specialized in it but they should be the one trying to motivate the rest of the staff to actually do it." (P9)

\section{DISCUSSION}

Pain assessment and management in older adults have inherent challenges, with significant implications for functional outcomes. ${ }^{1,2)}$ These challenges may be further complicated by other factors related to hospital-specific settings. ${ }^{3,4)}$ This study explored the views of clinicians in a tertiary hospital on the barriers and solutions for effective pain assessment and management. A painfree hospital project in Germany reported that more than half of the surgical and non-surgical patients were dissatisfied with pain management, with peak pain usually occurring outside normal working hours. ${ }^{9)}$ Therefore, it is important to integrate effective pain management into routine practice across ward settings, moving away from pain specialty teams toward a pain-free hospital.

Healthcare practitioners reported that a key barrier to effective pain management was a feeling of apprehension toward the appropriate use of diverse pain medications in acute hospital settings. The doctors and nurses who participated in this study may have had significant knowledge deficits and false beliefs that could impede their treatment of patients in pain. A study assessing the knowledge and attitudes of doctors and nurses found that only $61 \%$ of the questions were answered correctly, necessitating hospital-wide educational programs to improve clinicians' understanding of pain. ${ }^{10)}$ Reassessment of the impact of educational interventions in terms of pain assessment, patient satisfaction, and improvement in knowledge, as well as the provision of access to evidence-based resources to maintain updated knowledge on pain assessment and management, is also recommended. ${ }^{11,12)}$

Participants also shared concerns regarding under-treatment of pain due to inadequate knowledge of medications and the management of side effects or adverse reactions. A study implementing a pain treatment algorithm based on numerical pain ratings found that the rate of opioid adverse drug reactions doubled, especially over-sedation and decreased patient level of consciousness. ${ }^{13)}$ Dose adjustments and careful monitoring should be considered specifically for certain populations with higher risks, such as older patients, those with dementia, or those with previous cerebrovascular injuries. ${ }^{14)}$ These practical challenges recognized by the partici- pants in the present study should be addressed in educational sessions to avoid complications from pain management.

Our study also raised issues regarding patients' understanding of pain relief, which may impede effective pain management. A study from the United States reported that, despite improvements in pain treatment, the rate of moderate to severe postoperative pain remained $80 \%$, with more than half of patients expressing fear about pain after surgery. ${ }^{15)}$ Patient participation in decision-making regarding pain management is associated with less time in severe pain, better pain relief, lower pain severity, and improved quality of care. ${ }^{16)}$ These findings reinforce the need for increased professional and public awareness, including the establishment of pain management programs incorporating public and patient education. ${ }^{9}$

The results of our study also highlighted the need for a consistent clinical approach with collaboration and effective communication between staff. While pain management varies between hospitals and wards, the participants expressed a desire for a hospital culture conducive to optimizing pain control. A large Canadian teaching hospital reported worse pain management in non-surgical patients compared with that in surgical patients, with longer waiting times for medications, increased likelihood of prescribing ineffective medications, and the prescription of breakthrough pain relief when required in only half the medical patients. ${ }^{17)}$ Achieving a pain-free hospital requires the involvement of all health care teams to play important roles in acute pain medicine. These teams include physicians, nurses, pharmacists, psychologists, physiotherapists, occupational therapists, and patients. ${ }^{18)}$

There is also a narrative of hierarchy in hospitals, particularly where trainees are expected to not challenge or question the judgment or decisions of their seniors. This may be due to fears of appearing inadequate when facing uncertainty with complex patients or a desire to be viewed favorably due to their dependence on their seniors for career progression. ${ }^{19)}$ The power differentials between healthcare providers need to be acknowledged to develop models for shared responsibility between professions, particularly as a collaborative approach for pain management is necessary to improve patient outcomes. $^{20)}$

Educational activities and quality improvement initiatives should involve all medical providers to ensure a consistent clinical approach with a unified goal of providing safe, timely, and effective management of all acute pain scenarios. ${ }^{21)}$ The Toronto General Hospital Transitional Pain Service developed a multidisciplinary program to reduce the risk of postoperative pain, illustrating the benefits of a team approach and effective interprofessional communication. The comprehensive pain intervention addressed pain preoperatively, postoperatively, and at outpatient follow-up 6 months after surgery. Patients at high risk of pain complications were identi- 
fied early and their care coordinated by a team of pain physicians, advanced practice nurses, psychologists, and physiotherapists who used patient encounters as opportunities to impact pain trajectories. A clinical algorithm was used to provide clinical services through the different stages of surgical recovery, including preventative behavioral interventions to reduce the likelihood of pain. ${ }^{22)}$

Our study also identified cultural factors impacting pain assessment, including patients not requesting pain relief or with a high pain threshold in local settings. Pain assessment in older adults is challenging for multiple reasons, including multiple medical problems contributing to pain, an inability to self-report due to cognitive impairment, and the need to recognize atypical presentations or behavioral changes that may indicate pain. ${ }^{23)}$ In this setting, these challenges may also be exacerbated by pre-existing cultural beliefs, such as pain as an inevitable part of the human condition, societal attitudes toward pain relief during surgery and childbirth, pain seen as necessary or unavoidable, or associating chronic pain with psychological problems, resulting in a reluctance to request analgesia or medical attention. ${ }^{4)}$ The cultural validity of self-reported pain assessment tools should also be considered, and adjustments should be made to incorporate cultural views and tailor the language to suit the local population. ${ }^{24)}$ For example, words may have different meanings, confounding patients' understanding of their pain experience. Further work is required to establish shared meanings and pain descriptors due to the variety of languages spoken locally. ${ }^{25)}$

The participants also identified patients' preferences for non-pharmacological treatments. While this may be appropriate for initial treatment, an evidence-based approach is required. Clinicians must remain up-to-date on complementary and alternative approaches to provide patients information on these options for pain management. ${ }^{26)}$ Further study may be required to assess how culture impacts patient and clinician understanding of pain and to tailor pain assessment and management strategies to suit the target population.

The final theme discussed how to drive acute pain management forward through clinician buy-in and providing guidelines and mentorship. Guidelines developed by multidisciplinary panels of experts and based on the best available evidence are essential for promoting the effective and safe use of opioid therapy. ${ }^{27)}$ The development of pain practices may be promoted through nurse-tonurse mentoring and ongoing interactive case-based learning in pain management. ${ }^{28)}$ The Milan Cancer Institute, with extensive experience in pain assessment through validated tools, pain management, and educational efforts, also emphasized the importance of the long-term sustainability of these initiatives, with a need to persevere with continuing educational and informative programs to reduce pain frequency and severity and thus improve in-patient quality of life. $^{29)}$

The strengths of this study include its use of focus groups to gain insight into aspects of pain management from the main specialties dealing with clinical pain. However, the views of team members outside these specialties and allied health professionals may not have been fully represented. To move toward a pain-free setting, hospitals should focus on increased knowledge among clinicians and patients and a team approach to implement consistent pain management strategies that considers cultural factors to tailor plans to the local population. Future research should assess pain knowledge among clinicians and patients, the effectiveness of multidisciplinary pain management interventions, and the effects of culture on pain assessment and management.

In conclusion, the results of this study revealed clinicians' views of pain assessment and management in a tertiary hospital setting, including the strengths and limitations of the provision of acute pain service. Despite advances in pain medicine, effective pain management is proportionate to both clinical and cultural factors. These issues should be identified and resolved, as optimal pain management is required to avoid complications including dependence and loss of function in older adults. Future studies should investigate the standardization of global pain management tools and guidelines to fit the local culture and context.

\section{CONFLICT OF INTEREST DISCLOSURES}

The researchers claim no conflicts of interest.

\section{REFERENCES}

1. Reid MC, Eccleston C, Pillemer K. Management of chronic pain in older adults. BMJ 2015;350:h532.

2. Abdulla A, Adams N, Bone M, Elliott AM, Gaffin J, Jones D, et al. Guidance on the management of pain in older people. Age Ageing 2013;42 Suppl 1:i1-57.

3. Sinatra R. Causes and consequences of inadequate management of acute pain. Pain Med 2010;11:1859-71.

4. Brennan F, Carr DB, Cousins M. Pain management: a fundamental human right. Anesth Analg 2007; 105:205-21.

5. Gordon DB, Pellino TA, Miaskowski C, McNeill JA, Paice JA, Laferriere D, et al. A 10-year review of quality improvement monitoring in pain management: recommendations for standardized outcome measures. Pain Manag Nurs 2002;3:116-30.

6. Saifan AR, Bashayreh IH, Al-Ghabeesh SH, Batiha AM, Alrimawi I, Al-Saraireh M, et al. Exploring factors among healthcare professionals that inhibit effective pain management in cancer 
patients. Cent Eur J Nurs Midwifery 2019;10:967-76.

7. Milewska MM, Horosz B, Ladyko AR. Pain free hospital: recommendation for the acute pain management in Poland. J Pain Relief 2013;2:1000120.

8. Gordon K, Rice H, Allcock N, Bell P, Dunbar M, Gilbert S, et al. Barriers to self-management of chronic pain in primary care: a qualitative focus group study. Br J Gen Pract 2017;67:e209 -e217.

9. Maier C, Nestler N, Richter H, Hardinghaus W, Pogatzki-Zahn $\mathrm{E}$, Zenz M, et al. The quality of pain management in German hospitals. Dtsch Arztebl Int 2010;107:607-14.

10. Visentin M, Trentin L, de Marco R, Zanolin E. Knowledge and attitudes of Italian medical staff towards the approach and treatment of patients in pain. J Pain Symptom Manage 2001;22:92530.

11. Innis J, Bikaunieks N, Petryshen P, Zellermeyer V, Ciccarelli L. Patient satisfaction and pain management: an educational approach. J Nurs Care Qual 2004;19:322-7.

12. Medrzycka-Dabrowska W, Dabrowski S, Gutysz-Wojnicka A, Basinski A, Kwiecien-Jagus K. Nurses' knowledge and barriers regarding pain management. J Perianesth Nurs 2018;33:715-26.

13. Vila H Jr, Smith RA, Augustyniak MJ, Nagi PA, Soto RG, Ross TW, et al. The efficacy and safety of pain management before and after implementation of hospital-wide pain management standards: is patient safety compromised by treatment based solely on numerical pain ratings? Anesth Analg 2005;101:47480.

14. Lynch T. Management of drug-drug interactions: considerations for special populations: focus on opioid use in the elderly and long term care. Am J Manag Care 2011;17 Suppl 11:S293-8.

15. Warfield CA, Kahn CH. Acute pain management. Programs in U.S. hospitals and experiences and attitudes among U.S. adults. Anesthesiology 1995;83:1090-4.

16. Zoega S, Sveinsdottir H, Sigurdsson GH, Aspelund T, Ward SE, Gunnarsdottir S. Quality pain management in the hospital setting from the patient's perspective. Pain Pract 2015;15:236-46.

17. Sawyer J, Haslam L, Daines P, Stilos K. Pain prevalence study in a large Canadian teaching hospital. Round 2: lessons learned? Pain Manag Nurs 2010;11:45-55.

18. Schwenk ES, Baratta JL, Gandhi K, Viscusi ER. Setting up an acute pain management service. Anesthesiol Clin 2014;32:893-
910.

19. Crowe S, Clarke N, Brugha R. 'You do not cross them': hierarchy and emotion in doctors' narratives of power relations in specialist training. Soc Sci Med 2017;186:70-7.

20. Whitehead C. The doctor dilemma in interprofessional education and care: how and why will physicians collaborate? Med Educ 2007;41:1010-6.

21. Upp J, Kent M, Tighe PJ. The evolution and practice of acute pain medicine. Pain Med 2013;14:124-44.

22. Katz J, Weinrib A, Fashler SR, Katznelzon R, Shah BR, Ladak SS, et al. The Toronto General Hospital Transitional Pain Service: development and implementation of a multidisciplinary program to prevent chronic postsurgical pain. J Pain Res 2015;8: 695-702.

23. Bicket MC, Mao J. Chronic pain in older adults. Anesthesiol Clin 2015;33:577-90.

24. Booker SS, Herr K. The state-of-"cultural validity" of self-report pain assessment tools in diverse older adults. Pain Med 2015; 16:232-9.

25. Slater NG. The language of acute pain assessment: a corpus-based critical discourse analysis [dissertation]. Nottingham, UK: University of Nottingham; 2015.

26. Tick H, Nielsen A, Pelletier KR, Bonakdar R, Simmons S, Glick $\mathrm{R}$, et al. Evidence-based nonpharmacologic strategies for comprehensive pain care: the Consortium Pain Task Force white paper [Internet]. [place unknown]: www.nonpharmpaincare.org; c2017 [cited 2019 Dec 6]. Available from: http://dpbh.nv.gov/ uploadedFiles/dpbhnvgov/ content/Resources/opioids/Evidence_Based_Nonpharmacologic_Strategies_for_Comprehensive_Pain_Care_White_Paper.pdf.

27. Chou R, Fanciullo GJ, Fine PG, Adler JA, Ballantyne JC, Davies $\mathrm{P}$, et al. Clinical guidelines for the use of chronic opioid therapy in chronic noncancer pain. J Pain 2009;10:113-30.

28. De Witt Jansen B, Brazil K, Passmore P, Buchanan H, Maxwell D, Mcllfactrick SJ, et al. Nurses' experiences of pain management for people with advanced dementia approaching the end of life: a qualitative study. J Clin Nurs 2017;26:1234-44.

29. Ripamonti C, Zecca E, Brunelli C, Groff L, Boffi R, Caraceni A, et al. Pain experienced by patients hospitalized at the National Cancer Institute of Milan: research project "towards a pain-free hospital". Tumori 2000;86:412-8. 